\title{
Rupture Velocity of Plane Strain Shear Cracks
}

\author{
D. J. ANDREwS \\ U.S. Geological Survey. Menlo Park. California 94025
}

\begin{abstract}
Propagation of plane strain shear cracks is calculated numerically by using finite difference equations with second-order accuracy. The rupture model, in which stress drops gradually as slip increases, combines two different rupture criteria: (1) slip begins at a finite stress level; (2) finite energy is absorbed per unit area as the crack advances. Solutions for this model are nonsingular. In some cases there may be a transition from rupture velocity less than Rayleigh velocity to rupture velocity greater than shear wave velocity. The locus of this transition is surveyed in the parameter space of fracture energy, upper yield stress, and crack length. A solution for this model can be represented as a convolution of a singular solution having abrupt stress drop with a 'rupture distribution function.' The convolution eliminates the singularity and spreads out the rupture front in space-time. If the solution for abrupt stress drop has an inverse square root singularity at the crack tip, as it does for sub-Rayleigh rupture velocity, then the rupture velocity of the convolved solution is independent of the rupture distribution function and depends only on the fracture energy and crack length. On the other hand, a crack with abrupt stress drop propagating faster than the shear wave velocity has a lower-order singularity. A supershear rupture front must necessarily be spread out in space-time if a finite fracture energy is absorbed as stress drops.
\end{abstract}

\section{INTRODUCTION}

Theoretical predictions of the velocity of propagation of the tips of plane strain shear cracks (mode 2 cracks) appear contradictory. By one hypothesis it is agreed that if a finite fracture energy is absorbed per unit area of crack surface as the crack advances, then the rupture velocity must be less than the Rayleigh wave velocity [Kostrov, 1970; Freund, 1972; Fossum and Freund, 1975]. On the other hand, if slip begins when shear stress on the crack plane reaches a finite static friction level, then for a certain range of friction levels the rupture velocity cannot be less than the $P$ wave velocity [Burridge, 1973]. In both theoretical models, stress drops abruptly when slip starts, and the material is elastic off the crack plane. Stress drop arising from either fracture stick-slip friction is mathematically equivalent for motion in the medium, except for the criterion for propagation of the stress drop. In this paper the word rupture refers in general to the stress drop as slip begins.

In any real material there is a limit to the shear stress that the material can support, so that a solution with a stress singularity satisfying a fracture surface energy criterion must be modified in the neighborhood of the crack tip. On the other hand, a solution with rupture velocity chosen to give bounded shear stress on the crack plane will still have singularities at the crack tip in other components of shear stress due to the displacement having a singular derivative. Therefore even in the case of stick-slip friction, a region near the crack tip will be driven to its elastic limit and will absorb energy in excess of work done against the sliding friction stress [Andrews, 1976]. The energy absorbed need not be a material constant. A realistic rupture propagation criterion should combine both concepts, that shear stress be bounded and that the rupture front be a sink for energy of the elastic stress field.

Burridge, ignoring inelastic energy loss, concluded that molecular cohesive energy on a single ideal surface had a negligible effect at a length scale appropriate for an earthquake. Accordingly, he considered the limiting case of zero cohesive energy together with a finite stress limit. It is paradoxical that his conclusion regarding rupture velocity does not agree with the conclusion of fracture mechanics in the limit of vanishing fracture energy. The solution to the paradox, as we shall see in

Copyright (C) 1976 by the American Geophysical Union. the last section of this paper, is that stress drop is not abrupt. If both the finite stress and the finite fracture energy criteria apply, then energy is absorbed at the rupture front as inelastic deformation increases a finite amount driven by finite stress.

Rupture propagation at the $P$ wave velocity has been reported in a numerical calculation with a finite stress rupture criterion [Hamano, 1974]. In the present work, numerical calculations are performed to explore how the transition between sub-Rayleigh and super-Rayleigh rupture propagation depends on the three parameters, stress limit, fracture surface energy, and crack length.

\section{MODEL}

In this work, rupture propagation is calculated numerically with a uniform finite difference grid. In order that meaningful results may be obtained, a material model must be chosen for which the rupture front is nonsingular and is spread out in space enough to be adequately resolved by the finite difference grid. A particular idealized model is chosen that is characterized by an upper limit on shear stress on the crack plane and an effective fracture surface energy. Slip occurs only on a prescribed crack plane. The medium is infinite, homogeneous, isotropic, and linearly elastic off the crack plane. The rupture front is modeled with a slip-weakening law, in which shear stress on the crack drops gradually as slip (the discontinuity of displacement across the crack) increases. In this model, energy absorbed in inelastic strain near the crack tip is modeled as work done on the crack plane by the gradually decreasing stress.

Plane strain is assumed. Let the $y$ axis of a Cartesian coordinate system be normal to the crack plane. All variables are assumed to be independent of $z$, and the $z$ component of displacement is zero everywhere. Slip is in the $x$ direction. The possibility of inelastic coupling between antiplane strain and plane strain deformation at the rupture front [Richards, 1976] is disregarded.

In the slip-weakening model, a maximum traction $T$ at each point on the fault plane is prescribed as a function of the slip $\Delta u$ at that point. Traction is chosen to be a function of slip simply for convenience, so that fracture energy will be a constant of the model. In more realistic models, traction might be a function of slip velocity, as indicated by some friction experi- 
ments, and inelastic energy loss might occur in a zone of finite thickness governed by a strain-weakening law. In these cases, fracture energy is not a material constant, but if it is a slowly varying function of crack length, the present analysis will apply approximately. The slip-weakening model is specified as follows. When the crack is not slipping,

$$
\left|\tau_{x y}\right| \leq T(\Delta u) \quad \frac{\partial \Delta u}{\partial t}=0
$$

and during slip

$$
\tau_{x y}=T(\Delta u) \cdot \operatorname{sign}\left(\frac{\partial \Delta u}{\partial t}\right) \quad \frac{\partial \Delta u}{\partial t} \neq 0
$$

The slip-weakening model chosen (see Figure 1) is

$$
\begin{array}{ll}
T(\Delta u)=\tau_{u}-\left(\tau_{u}-\tau_{f}\right) \Delta u / d_{0} & \Delta u<d_{0} \\
T(\Delta u)=\tau_{f} & \Delta u \geq d_{0}
\end{array}
$$

where $\tau_{u}$ is the upper yield point, $\tau_{r}$ is the sliding friction level, and $d_{0}$ is the slip required for stress to drop. Ida [1972] has shown that this slip-weakening model leads to solutions with no singularity in antiplane strain, and it is expected that the same conclusion holds in plane strain. The inelastic work done at the rupture front in excess of the work done against the constant stress $\tau_{f}$ is divided between the two sides of the crack and identified as the effective fracture surface energy,

$$
G=\mathfrak{z}\left(\tau_{u}-\tau_{f}\right) d_{0}
$$

A further arbitrary assumption is that the reference state from which slip is measured is a state of uniform stress, $\tau_{x y}=$ $\tau_{0}$, throughout the medium. It is assumed that this ambient stress level lies between the upper yield point and the sliding friction level

$$
\tau_{u}>\tau_{0}>\tau_{f}
$$

If a crack propagates in this model, it will not stop. The ultimate size of real cracks is determined by nonuniformities of initial stress and material properties.

If initial slip is zero with respect to the arbitrary reference state, a rupture cannot nucleate. The reference state does not represent a fault just before an earthquake. The initial state that we choose for this model is a critical state, with nonuni-

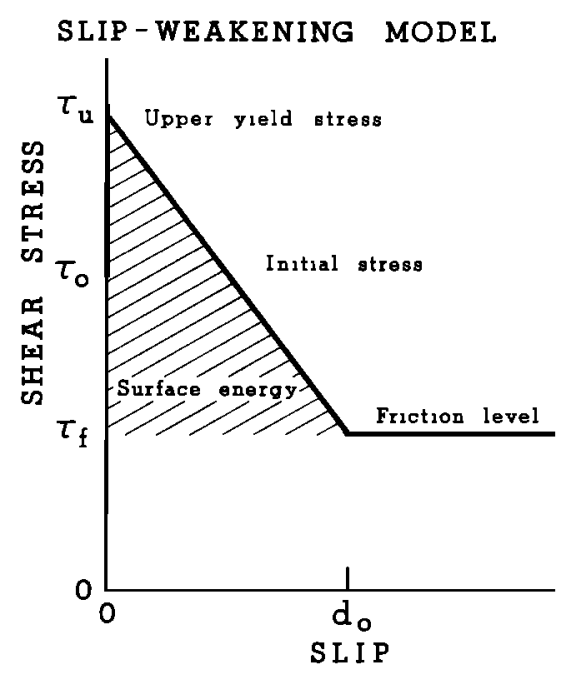

Fig. 1. Slip-weakening model. form stress and slip, which is on the verge of instability. The critical state is a generalization of a critical crack.

The ratio of the surface energy to the strain energy per unit volume available from the stress drop determines a characteristic length. With appropriate numerical factors the characteristic length is chosen to be the critical half-length of a Griffith crack in plane strain (derived in a later section of this paper),

$$
L_{c}=\frac{8}{\pi} \frac{\mu(\lambda+\mu)}{(\lambda+2 \mu)} \frac{G}{\left(\tau_{0}-\tau_{f}\right)^{2}}
$$

where $\lambda$ and $\mu$ are the Lamé constants. $L_{c}$ is the unit of length in the calculations, and $L_{c} / \beta$, where $\beta$ is the shear wave velocity, is the unit of time. Poisson's ratio is chosen to be 0.25 , so that $\lambda=\mu$, the $P$ wave velocity is $3^{1 / 2} \beta$, and the Rayleigh wave velocity is $0.9194 \beta$. All calculations are for bilaterally symmetric cracks in an infinite medium.

\section{Results}

The remaining adjustable parameter of the model is the ratio of the stress increase required to initiate slip to the final stress drop. The case $\left(\tau_{u}-\tau_{0}\right) /\left(\tau_{0}-\tau_{f}\right)=0.8$ is chosen for detailed examination. An approximate solution for the critical crack in this case is shown in Figure 2. It was found by a method used in earlier work in antiplane strain [Andrews, 1976]. In a critical state the strain energy released as the crack lengthens a small increment balances the energy absorbed in the region of the crack tip. The static stress solution for the slip function shown in Figure 2 lies about 2.5\% above the stress solution consistent with the slip-weakening relation. Therefore the slip function shown is slightly supercritical. This nearly critical solution is chosen as the initial state in the dynamic calculation in order to examine the smooth initial growth.

Rupture propagation in the dynamic solution evolving from the nearly critical solution is shown in Figure 3. The leading and trailing edges of the rupture front are plotted as two solid lines in the space-time plane. The leading edge is the locus separating zero from nonzero values of slip velocity, and the trailing edge is the locus $\Delta u=d_{0}$, where stress drop is complete.

Initial growth of a crack with singular tips is represented by a hyperbola in the $x$ - $t$ plane [Ida, 1972]. Initial growth in this calculation appears slower and may be exponential rather than quadratic in time.

The rupture is propagating at a significant fraction of the Rayleigh velocity by the time the crack has doubled its length and then approaches the Rayleigh velocity asymptotically. The width of the rupture front decreases as the crack lengthens. This behavior is consistent with that found in antiplane strain [Andrews, 1976], with the terminal velocity being the Rayleigh, rather than the shear wave, velocity.

Slices through the solution at a sequence of instants in time are shown in Figures 4-7, showing slip and shear stress on the crack plane as a function of position on the crack plane. A stress peak propagating ahead of the rupture front at the shear wave velocity gradually becomes higher, to the point where it starts driving some slip on the crack beyond $x=8 L_{c}$. Beyond $x=12 L_{c}$, the entire rupture front propagates faster than the shear wave velocity. The leading edge of rupture, shown in Figure 3, never propagates at a velocity between the Rayleigh and shear wave velocities. The rupture front is wider after the transition to higher velocity than before. After the transition the rupture velocity quickly goes to values greater than $1.5 \beta$.

In Figure 4 the rupture front is propagating at less than the Rayleigh velocity. The stress peak at the leading edge of rup- 


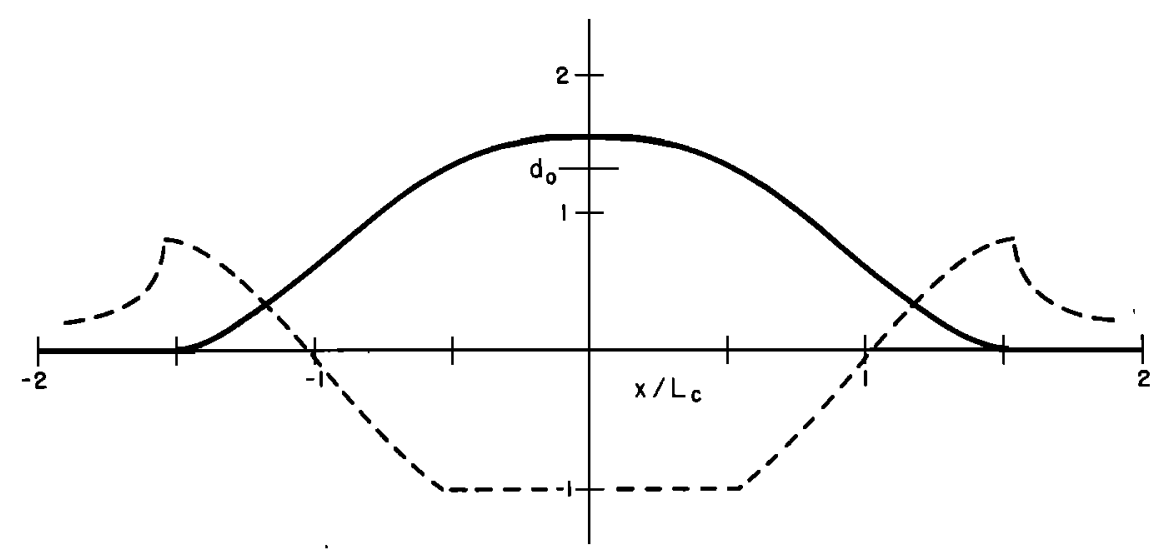

Fig. 2. Critical crack for the slip-weakening model with $\left(\tau_{u}-\tau_{0}\right) /\left(\tau_{0}-\tau_{f}\right)=0.8$. Solid curve is dimensionless slip function, $\mu \Delta u /\left[L_{c}\left(\tau_{0}-\tau_{f}\right)\right]$; dashed curve, dimensionless stress change, $\left(\tau_{x y}-\tau_{0}\right) /\left(\tau_{0}-\tau_{f}\right)$.

ture is preceded by another stress peak with smaller amplitude.

In an analytic solution for a self-similar crack with abrupt stress drop propagating at the Rayleigh velocity, Burridge [1973] predicts a finite stress peak at the shear wave front. The present numerical solution may be interpreted as such a selfsimilar solution smeared out in space-time to account for the finite rupture width and smeared out further to account for different initial growth. As the rupture propagates to greater lengths, the smearing out becomes relatively less important, so the stress peak at the shear wave front becomes more sharply defined and increases in amplitude.

The calculation was done with a grid spacing of $\Delta x=0.1 L_{c}$ to the time shown in Figure 4. In a second calculation going to a later time, $\Delta x=0.2 L_{c}$ was used.

In Figure 5 the stress peak at the shear wave front has reached the upper yield point. The amount of slip that has

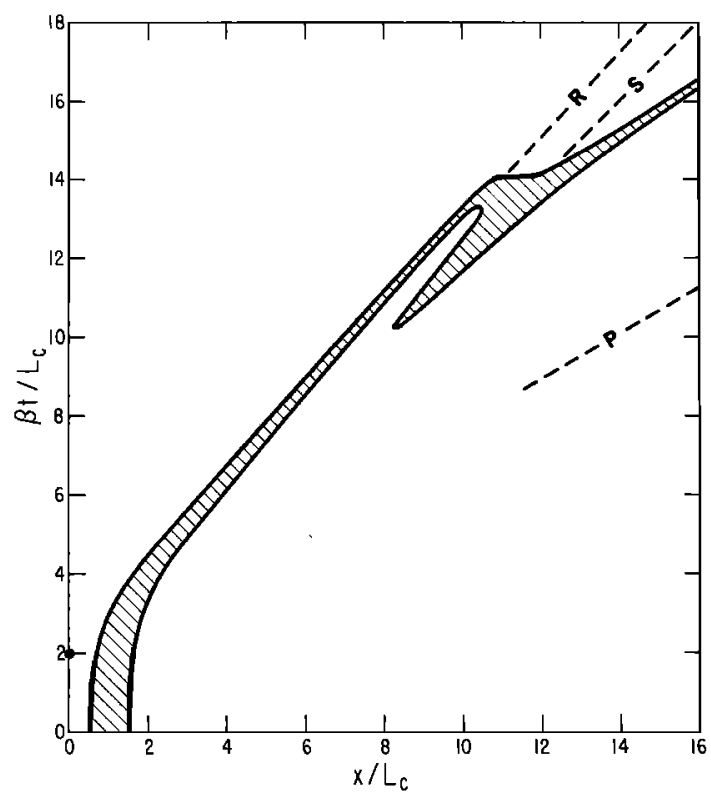

Fig. 3. Space-time plot of rupture propagation in dynamic calculation starting from nearly static solution of Figure 2. Region between the two solid lines is the rupture front, where slip velocity is nonzero and stress drop is incomplete. Dashed lines labeled $\mathbf{R}, \mathbf{S}$, and $P$, drawn for reference with slopes corresponding to Rayleigh, shear, and compressional wave velocities, respectively, diverge from the solid point on the time axis. been driven by this peak at this time is too small to show in the figure.

In Figure 6 the first arriving stress peak is broader, and the slip that it has driven is evident. The amount of slip at the second stress peak reduces the amplitude of that peak according to the slip-weakening relation. As this second stress peak propagates farther, it encounters larger values of slip and must attenuate more.

In Figure 7 the entire rupture front is propagating faster than the shear wave velocity.

In Figure 8 the slip velocity (discontinuity of particle velocity across the crack plane) is plotted as a function of position at the same instant of time as in Figure 6. Each point from the finite difference solution is shown. Point-to-point oscillation is generally suspect in any numerical calculation. The finite difference equations used in this work have no inherent damping for any wavelength, and no damping or smoothing operation, other than the slip-weakering law itself, has been added. Therefore the degree of smoothness of this solution is an indicator of its credibility (see appendix).

Other calculations were performed with the relative upper yield point parameter, $\left(\tau_{u}-\tau_{0}\right) /\left(\tau_{0}-\tau_{f}\right)$, having values 0.5 , 0.667 , and 1.0. In order to save computer time these calculations did not start from critical states but rather were started by forcing the stress drop to propagate at least as fast as $0.5 \beta$ in the computer calculation. From each calculation, two crack lengths were picked, marking the beginning and the com-

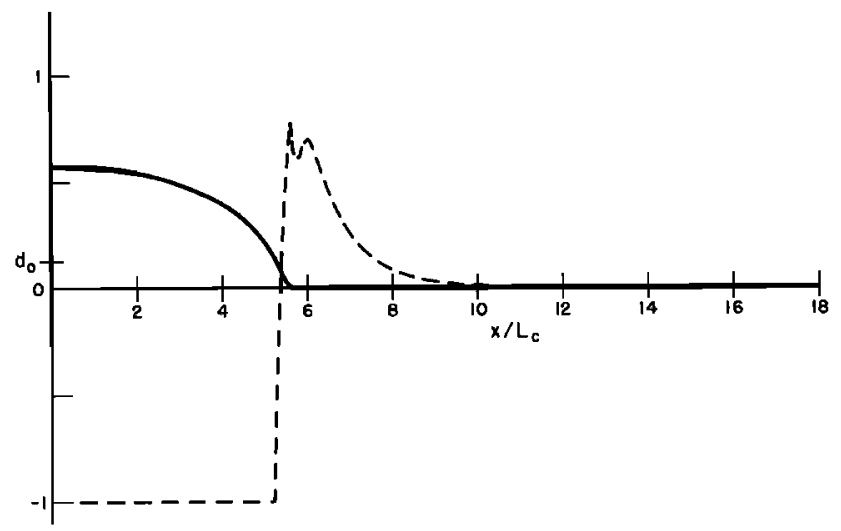

Fig. 4. Dynamic solution as a function of position on the crack plane at the dimensionless time $\beta t / L_{c}=8.07$. Solid curve is dimensionless slip function divided by $10, \mu \Delta u /\left[10 L_{c}\left(\tau_{0}-\tau_{f}\right)\right]$; dashed curve, dimensionless change of shear stress, $\left(\tau_{x y}-\tau_{0}\right) /\left(\tau_{0}-\tau_{f}\right)$. 


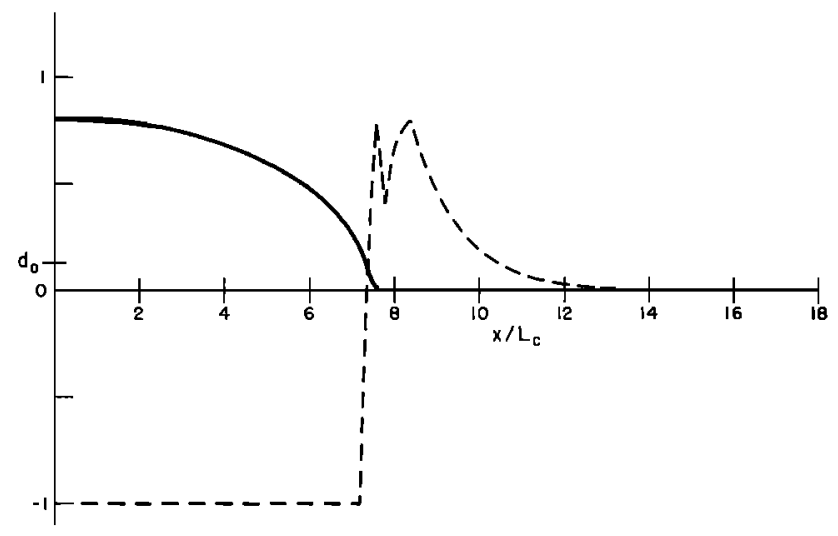

Fig. 5. Dynamic solution at $\beta t / L_{e}=10.38$. Legend is same as that for Figure 4.

pletion of the transition in rupture velocity. These picks, shown as the reciprocal of the ratio of length to critical length, are plotted as points in Figure 9. The points may be uncertain by about a factor of 2 . At small values of the relative upper yield point, the picks may be influenced by the starting procedure, and at larger values the rupture width is smaller and the calculation therefore less accurate. The shaded region in the figure is an interpretation of the transition region. It is drawn to intersect the horizontal axis at Burridge's predicted value for zero surface energy $\left(L_{c}=0\right)$.

\section{DisCUSSION OF RESULTS}

The length ratio plotted in Figure 9 is equivalent to the ratio of energy absorbed at the rupture front to available strain energy released from a static crack of the same length. For a static crack with length $L$ and singular tips, the slip function is [Starr, 1928]

$$
\Delta u=\frac{\lambda+2 \mu}{\lambda+\mu} \frac{\tau_{0}-\tau_{f}}{\mu}\left(L^{2}-x^{2}\right)^{1 / 2}
$$

A dynamic crack propagating slower than the Rayleigh velocity has the same shape [Burridge and Willis, 1969]. The slip function in Figure 4 is about 0.73 times the slip function for a static crack with the same length and stress drop. The virtual work done in creating the static crack is

$$
U=-\frac{1}{2}\left(\tau_{0}+\tau_{f}\right) \int \Delta u d x
$$

and the work done against friction is

$$
W=\tau_{f} \int \Delta u d x
$$

As the crack lengthens by an increment $d L$, the energy available to be radiated away and to supply surface energy to extend the crack is

$$
-d U-d W=\frac{\pi}{2} \frac{\lambda+2 \mu}{\mu(\lambda+\mu)}\left(\tau_{0}-\tau_{f}\right)^{2} L d L
$$

The energy absorbed at both ends and on both sides of the crack is $4 G d L$.

The crack length at which the available energy released equals the energy absorbed is by definition the critical length. Equating the two expressions above, we find

$$
L_{c}=\frac{8}{\pi} \frac{\mu(\lambda+\mu)}{(\lambda+2 \mu)} \frac{G}{\left(\tau_{0}-\tau_{f}\right)^{2}}
$$

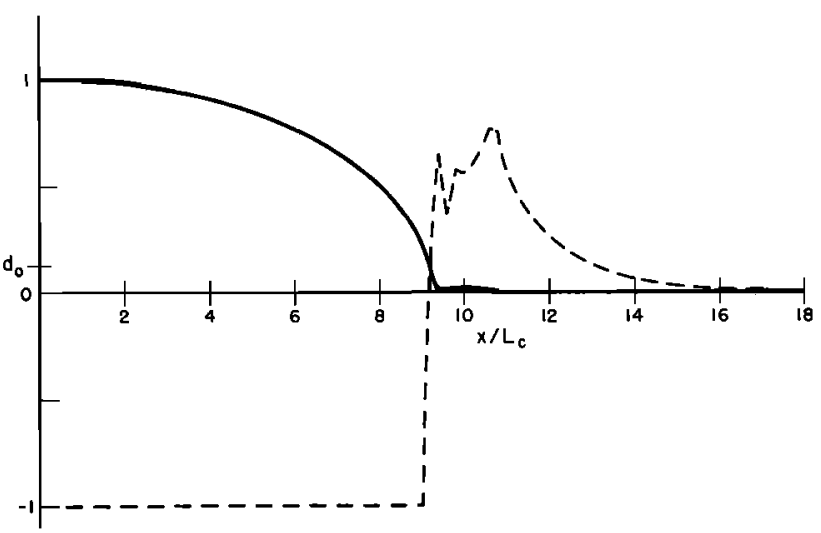

Fig. 6. Dynamic solution at $\beta t / L_{c}=12.36$. Legend is same as that for Figure 4.

It follows that in general the ratio of energy absorbed to available energy released is $L_{c} / L$.

These calculations have been performed with constant values of fracture surface energy. This assumption is not realistic at a large length scale (as for an earthquake) or in any case in which the energy is absorbed in inelastic strain distributed through a volume around the crack tip. If the energy absorption is governed by an inelastic stress-strain relation, then the thickness of the inelastic volume will be proportional to crack length, and $G$ and $L_{c}$ will be proportional to $L$. A rupture propagating at constant stress drop will tend toward a constant value of $L_{c} / L$ as $L$ increases. In an antiplane strain calculation with parameters chosen to give large energy absorption, the value $L_{c} / L=0.3$ was found [Andrews, 1976]. Smaller values can be expected in general, particularly for stick-slip friction on a preexisting fault.

The amplitude of the slip function of a propagating crack and stress intensity at the crack tip are determined primarily by stress drop, crack length, and instantaneous crack velocity. Variation arising from dependence on past history of rupture propagation will be less than a factor of 2 for the smooth variation considered here. Therefore within a factor of 2 , Figure 9 will obtain for cases with constant $L_{c} / L$, and each calculation has sampled crack behavior through a range of values of $L_{c} / L$.

\section{Theoretical Discussion}

Some insight into the rupture process may be gained by relating two different general approaches to modeling sources in an otherwise linear medium. One approach starts with a constitutive relation for the medium (which will be nonlinear in the source region) and deals with the system of partial differential equations that represent the interaction of each infinitesimal volume element with its neighbors. The finite difference calculations discussed in this paper are approximate solutions found following this first approach.

A second general approach starts with a Green's function, a solution to the linear problem for a point source. More general solutions are represented by convolutions of source distribution functions with the Green's function. A source distribution function can be judged to be physically realistic by the degree to which stress and strain in the convolved solution are in accord with a realistic material response. A constraint on admissible material response, such as a bound on stress, constrains the class of admissible source distributions.

For example, the Green's function representing a point double couple, convolved with an elliptical slip function, is the 


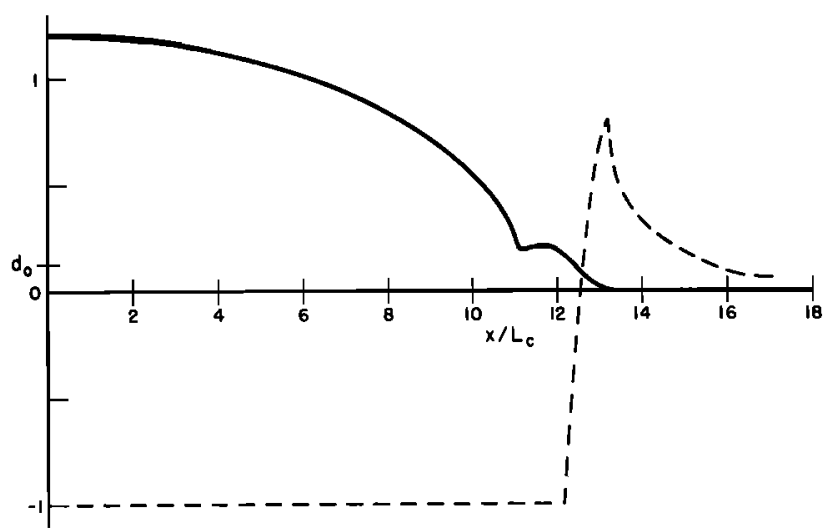

Fig. 7. Dynamic solution at $\beta t / L_{c}=14.34$. Legend is same as that for Figure 4.

solution for a shear crack. Such a solution is appropriate to the constitutive relation that stress on the crack is uniform for any nonzero value of slip.

If a solution for a crack with singular tips is further convolved with a function, $f(t)$, called here the 'rupture distribution function,' the crack tip can be smeared out to become a smooth rupture front. If the domain on which $f(t)$ has nonzero values is small, the extent of the smearing out is small, and there is still uniform stress on the slipped region in the largescale solution. To preserve the normalization of the large-scale solution, we require

$$
\int_{-\infty}^{\infty} f(t) d t=1
$$

To each rupture distribution there corresponds a constitutive relation, which may or may not be realistic. As the crack grows, it may be necessary that the rupture distribution function change to maintain the same constitutive relation.

Response of real materials during rupture is generally unknown. If stress is bounded, then inelastic work must be done in a finite deformation to yield a finite fracture energy; the transition from the elastic to the fractured state cannot be abrupt. A smeared out rupture front is reasonable. Any conclusion regarding rupture propagation that holds for a general

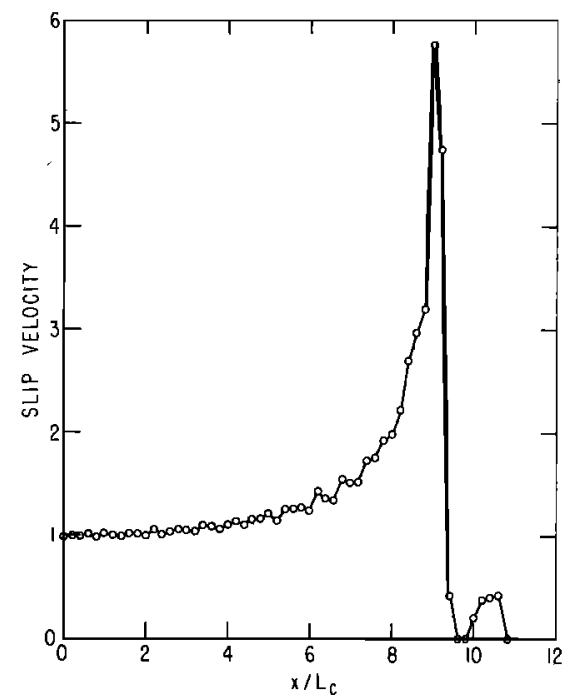

Fig. 8. Dimensionless slip velocity, $\mu(\partial \Delta u / \partial t) /\left[\beta\left(\tau_{0}-\tau_{f}\right)\right.$, as a function of position on the crack in the dynamic solution at $\beta t / L_{c}=12.36$.

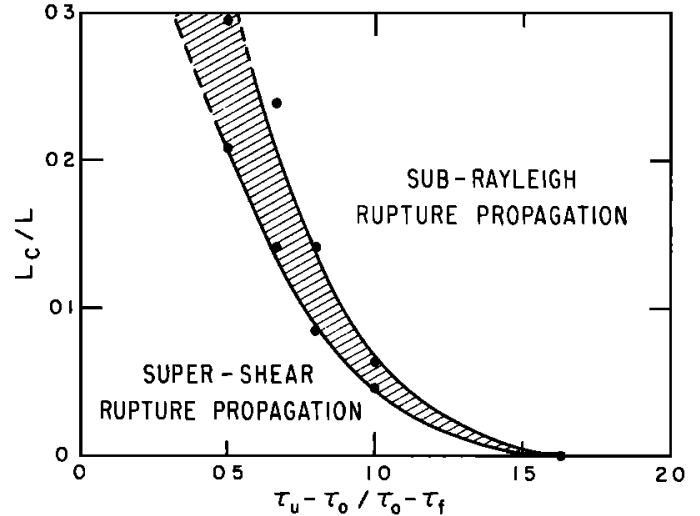

Fig. 9. Rupture velocity domains in parameter space. Shaded region is transitional. Vertical axis is ratio of critical length to crack length; horizontal axis is ratio of stress increase required to initiate slip to final stress drop.

class of rupture distributions can be expected to hold for a general class of constitutive relations.

The convolution of a singular crack solution with a rupture distribution function is a valid elastodynamic solution everywhere off the crack plane. The crack tip is smeared out into a rupture front region on the crack plane. Stress, $\tau_{x y}$, and particle velocity on each side of the crack, $\partial u_{+} / \partial t$ and $\partial u_{-} / \partial t$, in the convolved solution are continuous functions of time, $t$, and position on the crack plane, $x$, for a rupture distribution function that is piecewise continuous. Are these quantities continuous with the solution off the crack plane? Assume that the singular solution is a continuous function of $y$, the coordinate normal to the crack plane, in the sense of distribution theory [Zemanian, 1965, pp. 36, 72], meaning that averages over any finite interval of $x$ or $t$ are continuous functions of $y$. If this conjecture is true, then the convolved solution is a continuous function of $y$ as the crack plane is approached from either side, by the continuity property of convolution [Zemanian, 1965, p. 135].

Then the product $\tau_{x y} \partial \Delta u / \partial t$ in the rupture front on the crack plane represents the flux of energy to the crack plane from the stress field off the crack plane.

In order to resolve the paradox discussed in the introduction, the question of energy absorption at the rupture front for supershear rupture velocity needs to be examined. For an abrupt stress drop propagating faster than the shear wave speed, Burridge [1973] predicts a stress singularity of smaller order than the inverse square root singularity of slower cracks. We will see that such a crack tip can absorb energy only if it is smeared out in space-time.

Let the arrival time of the crack tip at $x=L$ be $t=t_{0}$. Near the arrival time the self-similar solutions obtained by Burridge for both sub-Rayleigh and supershear rupture velocity can be put in the form

$$
\begin{gathered}
\tau_{x y}(L, t)=K(v) \Delta \tau\left[v\left(t_{0}-t\right) / L\right]^{-a} \\
\cdot H\left(t_{0}-t\right)-\Delta \tau H\left(t-t_{0}\right) \\
\Delta u(L, t)=u_{+}-u_{-}=Q(v)(L \Delta \tau / \mu) \\
\cdot\left[v\left(t-t_{0}\right) / L\right]^{1-a} H\left(t-t_{0}\right) \\
\frac{\partial \Delta u(L, t)}{\partial t}=(1-a) Q(v)(v \Delta \tau / \mu)\left[v\left(t-t_{0}\right) / L\right]^{-a} H\left(t-t_{0}\right)
\end{gathered}
$$

where $H$ is the Heaviside step function, $\Delta \tau$ is the initial minus 
the sliding friction stress, and $v$ is rupture velocity. For $v$ less than the Rayleigh velocity, the exponent of the crack tip singularity is $a=\frac{1}{2}$; for $v \geq \beta$ we will see below that $0 \leq a \leq \frac{1}{2}$. These equations have been written to make clear the dimensional scaling appropriate for a crack growing in an infinite uniform medium. The functions $K$ and $Q$ are dimensionless and depend on rupture velocity alone. $K$ is not the conventional stress intensity factor, for the dependence on stress drop and crack length has been factored out. Explicit expressions for $K$ and $Q$ might be found by extending Burridge's analysis but are not needed here. The stress singularity does not overlap the slip function.

For the purpose of illustration, let the rupture distribution function be a square pulse,

$$
f(t)=(2 \epsilon)^{-1} H(\epsilon+t) H(\epsilon-t)
$$

and find the convolved stress and slip velocity

$$
\begin{aligned}
\left\langle\tau_{x y}(t)\right\rangle & =\int f\left(t^{\prime}\right) \tau_{x y}\left(t-t^{\prime}\right) d t^{\prime} \\
\left\langle\frac{\partial \Delta u(t)}{\partial t}\right\rangle & =\int f\left(t^{\prime}\right) \frac{\partial \Delta u\left(t-t^{\prime}\right)}{\partial t} d t^{\prime}
\end{aligned}
$$

The result can be expressed in three different time domains as follows. If $t<t_{0}-\epsilon$,

$$
\begin{aligned}
\left\langle\tau_{x y}(t)\right\rangle= & \frac{K(v) \Delta \tau}{(1-a)} \frac{L}{2 \epsilon v}\left\{\left[v\left(t_{0}-t+\epsilon\right) / L\right]^{1-a}\right. \\
& \left.-\left[v\left(t_{0}-t-\epsilon\right) / L\right]^{1-a}\right\} \\
\left\langle\frac{\partial \Delta u(t)}{\partial t}\right\rangle & =0 \\
\text { If } t_{0}-\epsilon<t<t_{0}+\epsilon, & -\frac{\Delta \tau}{2 \epsilon}\left(t-t_{0}+\epsilon\right) \\
\left\langle\tau_{x y}(t)\right\rangle= & \frac{K(v) \Delta \tau}{(1-a)} \frac{L}{2 \epsilon v}\left[v\left(t_{0}-t+\epsilon\right) / L\right]^{1-a} \\
\left\langle\frac{\partial \Delta u(t)}{\partial t}\right\rangle & =Q(v) \frac{v \Delta \tau}{\mu} \frac{L}{2 \epsilon v}\left[v\left(t-t_{0}+\epsilon\right) / L\right]^{1-a} \\
\text { If } t>t_{0}+\epsilon, & \\
\left\langle\tau_{x v}(t)\right\rangle & =-\Delta \tau \\
\left\langle\frac{\partial \Delta u(t)}{\partial t}\right\rangle & =Q(v) \frac{v \Delta \tau}{\mu} \frac{L}{2 \epsilon v}\left\{\left[v\left(t-t_{0}+\epsilon\right) / L\right]^{1-a}\right.
\end{aligned}
$$

The peak value of stress occurs at $t=t_{0}-\epsilon$ :

$$
\max \left\langle\tau_{x y}(t)\right\rangle=\frac{K(v) \Delta \tau}{(1-a)}\left(\frac{2 \epsilon v}{L}\right)^{-a}
$$

In the region where the stress and velocity peaks overlap, $t_{0}-\epsilon$ $<t<t_{0}+\epsilon$, stress is a decreasing function of time, and slip is increasing, as in some slip-weakening model. Different rupture distribution functions will represent different slip-weakening models.

With a finite overlap of finite functions the energy absorption is determined straightforwardly. We want to calculate the work done per unit area by the excess of stress over the final stress.

$$
\begin{aligned}
2 G=\int\left\langle\tau_{x y}(t)\right. & +\Delta \tau\rangle\left\langle\frac{\partial \Delta u(t)}{\partial t}\right\rangle d t \\
= & \frac{K(v) Q(v) L}{(1-a)} \frac{(\Delta \tau)^{2}}{\mu}(2 \epsilon)^{-2}(v / L)^{1-2 a} \\
& \cdot \int_{t_{0}-\epsilon}^{t_{0}+\epsilon}\left(t_{0}-t+\epsilon\right)^{1-a}\left(t-t_{0}+\epsilon\right)^{1-a} d t \\
+ & Q(v) L \frac{(\Delta \tau)^{2}}{\mu}(2 \epsilon)^{-2}(v / L)^{1-a} \\
& \cdot \int_{t_{0}-\epsilon}^{t_{0}+\epsilon}\left(t_{0}-t+\epsilon\right)\left(t-t_{0}+\epsilon\right)^{1-a} d t
\end{aligned}
$$

The substitution $s=\left(t-t_{0}\right) / \epsilon$ removes the dependence on $\epsilon$ from the integrals. Then

$$
\begin{aligned}
2 G & =\frac{K(v) Q(v) L}{4(1-a)} \frac{(\Delta \tau)^{2}}{\mu}\left(\frac{v \epsilon}{L}\right)^{1-2 a} \\
& \cdot \int_{-1}^{+1}(1-s)^{1-a}(1+s)^{1-a} d s \\
+ & \frac{Q(v) L}{4} \frac{(\Delta \tau)^{2}}{\mu}\left(\frac{v \epsilon}{L}\right)^{1-a} \int_{-1}^{+1}(1-s)(1+s)^{1-a} d s
\end{aligned}
$$

For small $\epsilon$ the first term will dominate the second.

Peak stress and $G$ are characteristic of the constitutive relation. If the shape of the slip-weakening curve is consistent with a square pulse rupture distribution, then (2) and (3) are a pair of simultaneous equations that determine $\epsilon$ and $v$. If $G / L$ is constant, then the solution is self-similar with $\epsilon$ proportional to $L$ and $v$ constant. In that case this analysis is rigorous. Otherwise, $v$ will vary with $L$, and values of $K$ and $Q$ from a self-similar analysis will not apply exactly.

Other shapes of the rupture distribution function can be considered. Assume that $f(t)$ is normalized according to (1), that it is zero outside a bounded domain, and that it is piecewise continuous. Then the one-parameter family of rupture distribution functions $(1 / \epsilon) f(t / \epsilon)$ satisfies the same conditions. Then expressions found for peak stress and fracture energy in the convolved solution, analogues of (2) and (3), will depend on the rupture width $\epsilon$. The two equations together will determine $\epsilon$ and $v$. The solution will depend on the choice of shape of the rupture distribution function $f(t)$.

From this point we will assume that $\epsilon$ is small enough that only the first term of (3), the term arising from the overlap of the two smeared out singularities, need be considered. This assumption may require that peak stress be large. Then if $a=$ 1 , as for sub-Rayleigh rupture velocity, some special properties apply. The dependence on $\epsilon$ disappears from (3). Then rupture velocity is determined by (3) alone and is independent of the extent of smearing out that is required to satisfy the stress limit.

For $a=\frac{1}{2}$, rupture velocity is also independent of the shape of the rupture distribution function. In this case the fracture energy, calculated from the overlap of the singularities, is

$$
2 G=\frac{K(v) Q(v) L(\Delta \tau)^{2}}{2 \mu} I
$$

where

$$
\begin{aligned}
I= & \iiint f\left(t^{\prime}\right)\left(t^{\prime}+t_{0}-t\right)^{-1 / 2} H\left(t^{\prime}+t_{0}-t\right) \\
& \cdot f\left(t^{\prime \prime}\right)\left(t-t_{0}-t^{\prime \prime}\right)^{-1 / 2} H\left(t-t_{0}-t^{\prime \prime}\right) d t^{\prime} d t^{\prime \prime} d t
\end{aligned}
$$


Let $s=t-t_{0}$. Then

$$
\begin{aligned}
I=\int_{-\infty}^{\infty} d s \int_{0}^{\infty} d s^{\prime} \int_{-\infty}^{s} d s^{\prime \prime} \\
\quad \cdot\left[f\left(s^{\prime}\right) f\left(s^{\prime \prime}\right)\left(s^{\prime}-s\right)^{-1 / 2}\left(s-s^{\prime \prime}\right)^{-1 / 2}\right]
\end{aligned}
$$

Note that the domain of integration is restricted to $s^{\prime}>s^{\prime \prime}, s^{\prime}>$ $s$, and $s>s^{\prime \prime}$, and change the order of integration

$$
\begin{aligned}
I=\int_{-\infty}^{\infty} d s^{\prime} \int_{-\infty}^{a^{\prime}} d s^{\prime \prime} f\left(s^{\prime}\right) f\left(s^{\prime \prime}\right) & \\
& \cdot \int_{a^{\prime \prime}}^{a^{\prime}} d s\left(s^{\prime}-s\right)^{-1 / 2}\left(s-s^{\prime \prime}\right)^{-1 / 2}
\end{aligned}
$$

The last integral is simply $\pi$. Let

$$
F(s)=\int_{-\infty}^{s} f\left(s^{\prime}\right) d s^{\prime}
$$

Then

$$
\begin{aligned}
& I=\pi \int_{-\infty}^{\infty} d s^{\prime} f\left(s^{\prime}\right) \int_{-\infty}^{s^{\prime}} d s^{\prime \prime} f\left(s^{\prime \prime}\right)=\pi \int F d F \\
& \quad=(\pi / 2)\left[F^{2}(s=+\infty)-F^{2}(s=-\infty)\right]=\pi / 2
\end{aligned}
$$

so that

$$
2 G=(\pi / 4) K(v) Q(v) L(\Delta \tau)^{2} / \mu
$$

The same result for energy absorption may be found for the case of a singular crack with $a=\frac{1}{2}$ by considering the Fourier transforms of $\tau_{x y}$ and $\partial \Delta u / \partial t$ and applying Parseval's relation to their inner product [Freund, 1972; Richards, 1976]. The reason that it is physically realistic to deal with singular cracks in the case $a=\frac{1}{2}$ is that rupture velocity is independent of the rupture distribution function, so that only the Dirac delta function need be considered. If $a \neq 1$, we must retreat from this level of abstraction.

If $v>\beta$, the exponent $a$ of the singularity at the crack tip is determined by the phase of the Rayleigh function with the rupture velocity $v$ as its argument [Burridge, 1973],

$$
R(v)=(-1)^{a}|R(v)|
$$

where

$$
R(v)=\left[1-v^{2} /\left(2 \beta^{2}\right)\right]^{2}-\left(1-v^{2} / \beta^{2}\right)^{1 / 2}\left(1-v^{2} / \alpha^{2}\right)^{1 / 2}
$$

and $\alpha$ is $P$ wave velocity. On the appropriate branch of the Rayleigh function,

$$
0 \leq a \leq \frac{1}{2}
$$

Burridge's analysis also allows other values of $a$, larger by any integer. These values are excluded by the requirement that energy be finite. The value of $a$ increases from zero at $v=\beta$ to $\frac{1}{2}$ at $v=2^{1 / 2} \beta$ and then decreases to zero at $v=\alpha$.

With $a<\frac{1}{2}$ there is no energy absorption for $\epsilon=0$, by (3). If fracture energy is nonzero, then (2) and (3) together determine $\epsilon$ and $v$, and $\epsilon$ will be nonzero.

The solution to the paradox is that for supershear rupture propagation with energy absorption, the stress drop cannot be abrupt.

Released strain energy is radiated preferentially in the direction of rupture propagation. For rupture velocity less than the Rayleigh velocity, a portion of the released energy can be focused on a point, the rupture front, and is absorbed there. For rupture velocity greater than the shear wave velocity, a point focus is not possible, but energy can still be delivered to a distributed rupture front.

After the completion of the transition in the numerical calculations, the rupture velocity quickly goes to values larger than $2^{1 / 2} \beta$, the domain where $a$ is a decreasing function of $v$. It is a reasonable conjecture that stable values of rupture velocity are confined to the domains $0<v<v_{R}$ and $2^{1 / 2} \beta<v<\alpha$, where $v_{R}$ is Rayleigh velocity.

The condition for the transition from sub-Rayleigh to supershear rupture velocity might be found analytically. For a particular choice of shape of rupture distribution function, the question can be posed as follows. When a self-similar solution for sub-Rayleigh rupture velocity is smeared out to reduce stress at the crack tip to the upper yield point, is stress at the $S$ wave peak above or below the upper yield point?

The finite difference method is not well suited to problems having two length scales that are greatly different. For values of rupture front length that are smaller fractions of crack length than considered here, convolution of analytic solutions would be a preferable method.

The slip-weakening model is not entirely realistic but is itself an abstraction of a strain-weakening model, in which failure takes place in a volume of finite thickness. Numerical solutions for a strain-weakening model [Andrews, 1976] resemble a crack solution smeared out in the direction normal to the crack plane, as well as on the crack plane. The rupture distribution function would have nonzero values over a finite inter$\mathrm{val}$ in $y$, as well as in $t$ or $x$. To establish that such a convolved solution is valid, it would be necessary to show that the correct equation of motion is satisfied within the rupture front volume and that only the constitutive relation is changed. I have no proof of this conjecture.

The ideas discussed in this section have been arrived at largely by induction. Concepts from distribution theory [ $\mathrm{Ze}$ manian, 1965] have been helpful to me in establishing to some extent a logical foundation for the ideas. In this point of view a singular crack solution is regarded as a distribution functional, and physical solutions are regularizations of it defined by convolutions with testing functions.

\section{Conclusions}

In real materials a rupture propagation criterion should require both that shear stress be bounded and that energy be absorbed at the rupture front. For a rupture propagating slower than the Rayleigh wave speed, only the energy absorption enters into the determination of rupture velocity, and the smearing out needed to satisfy the stress limit leaves the former relation unchanged. However, both criteria enter into the determination of whether a plane strain shear crack will propagate faster than the shear wave velocity. In that case, the rupture front cannot be singular but must be spread out in space-time.

Numerical calculations have been performed with a particular model combining both rupture criteria. The only kinematic assumption of the work is that rupture be restricted to a plane surface. Rupture velocity domains have been surveyed in the parameter space of upper yield point and ratio of energy absorbed to energy released.

What is the rupture velocity of an earthquake? The question will be best answered from observations. A predictive calculation of rupture velocity in an actual case would require detailed specification of material response during failure, for both the fault zone and the surrounding rock. Such properties are poorly understood. And in an actual case, stress drop will not be uniform as in the idealized model considered here. 


\section{APPENDIX: Numerical ACCURACY}

The numerical method used in this work has been reported previously [Andrews, 1973]. The spatial differencing is equivalent to uniform strain triangular finite elements, and the explicit time stepping is equivalent to the leapfrog scheme [Richtmyer and Morton, 1967].

Naive attempts to calculate crack propagation numerically are quite vulnerable to error. For instance, I once experimented 'with rupture calculations with abrupt stress drop triggered at a finite stress level. In some cases the initial crack would not propagate at all, for the stress concentration was not resolved well enough by the grid. In other cases the rupture would advance one grid point in every time step, faster than the $P$ wave velocity. Although those results could be changed with various averaging procedures at the crack tip, they demonstrate that numerical çrack calculations will not be credible without an understanding, first, of singularities to be expected for the problem as posed, second, of reasonable physical mechanisms that will remove the singularities, and, third, of the characteristics of the numerical system itself. Special procedures to match an expected singularity to a numerical solution could introduce unexpected complications and would require some kinematic assumptions. Therefore I chose a physical model for which no singularity is expected and used a simple numerical procedure having properties that are well understood.

The essential characteristics of the numerical system can be illustrated in one dimension with a uniform material and a uniform grid. The one-dimensional linear scalar wave equation can be represented by the pair of coupled equations

$$
\frac{\partial v}{\partial t}=\frac{1}{\rho} \frac{\partial \sigma}{\partial x} \quad \frac{\partial \sigma}{\partial t}=\rho c_{n}^{2} \frac{\partial v}{\partial x}
$$

where $v$ is particle velocity, $\sigma$ is stress, $\rho$ is density, and $c_{0}$ is wave speed. In the finite difference equations, a subscript $j$ indicates the point in space, $x=j \Delta x$; a superscript $n$, the point in time, $t=n \Delta t$. The leapfrog finite difference scheme is

$$
\begin{gathered}
v_{i}{ }^{n+1 / 2}-v_{i}{ }^{n-1 / 2}=\frac{\Delta t}{\rho \Delta x}\left(\sigma_{j+1 / 2}{ }^{n}-\sigma_{i-1 / 2}{ }^{n}\right) \\
\sigma_{i+1 / 2}{ }^{n+1}-\sigma_{i+1 / 2}{ }^{n}=\frac{\rho c_{0}{ }^{2} \Delta t}{\Delta x}\left(v_{i+1}{ }^{n+1 / 2}-v_{i}{ }^{n+1 / 2}\right)
\end{gathered}
$$

With these equations the solution can be stepped through time explicitly. If the time step does not satisfy the stability condition

$$
\Delta t \leq \Delta x / c_{0}
$$

the shortest wavelength component of the solution will grow exponentially. As instability in a solution will be obvious, it is more important to understand accuracy.

Accuracy of the finite difference equations can be expressed in terms of impedance, dissipation, and dispersion as a function of wavelength for a sinusoidal wave. Assume

$$
\begin{aligned}
& v=v_{0} \exp i(k x-\omega t) \\
& \sigma=\sigma_{0} \exp i(k x-\omega t)
\end{aligned}
$$

and substitute into the finite difference equations. One finds, first, that
The impedance is therefore correct for all wavelengths. Second, if the stability condition is met, then $k$ and $\omega$ are both real, and there is no dissipation. Third, $k$ and $\omega$ are related by the dispersion relation

$$
\sin (k \Delta x / 2)=\Delta x /\left(c_{0} \Delta t\right) \sin (\omega \Delta t / 2)
$$

To second order, the phase velocity is

$$
c / c_{0}=\omega /\left(k c_{0}\right)=1-\frac{1}{6}\left[1-\left(\frac{c_{0} \Delta t}{\Delta x}\right)^{2}\right]\left(\frac{k \Delta x}{2}\right)^{2}
$$

At the stability limit, $c_{0} \Delta t / \Delta x=1$, the truncation error in time differences cancels the truncation error in space differences. However, dispersion cannot be avoided in this way in two dimensions, for the effective parameter $c_{0} \Delta t / \Delta x$ is not the same in different directions, nor is it the same for $S$ waves and $P$ waves. For small $\Delta t$ the phase velocity for 2 points per wavelength is $c / c_{0}=0.638$; for 4 points per wavelength, 0.902 .

The dispersion relation for a two-dimensional grid has been worked out by Alford et al. [1974]. In the symmetry directions of the grid the result is the same as the one-dimensional case, and this will be true for any second-order generalization to two space dimensions. The equations of Madariaga [1976] are more efficient than mine in storage requirements and number of operations, but the dispersion relation is the same.

Implicit equations, for which there may be no stability requirement, provide no significant advantage in accuracy.

For a linear problem, different wavelengths are not coupled, so short-wavelength noise is irrelevant. It can be either filtered out or ignored. In the present work a nonlinear boundary condition is used on the crack surface, and short-wavelength components can affect the rupture propagation. In order that a numerical solution be accurate, it is necessary that the correct solution for the assumed physical model be smooth enough that amplitudes of short-wavelength components are not significant. For a rupture width of $4 \Delta x$, the shortest significant wavelength would be about $8 \Delta x$, and the relative error would be less than $2 \%$ for all components. Unfortunately, the minimum rupture width in this work is only $\Delta x$. The grid size used was $100 \times 200$, and it would be quite expensive to go to finer resolution. Two calculations, one with $\Delta x=0.1 L_{c}$, the other with $\Delta x=0.2 L_{c}$, agreed reasonably well and showed similar stress peaks at shear wave front ahead of the rupture. The calculation with finer resolution did not go far enough for the transition in rupture velocity to occur.

Dispersion will tend to reduce peak values of stress and particle velocity. Therefore the transition to higher rupture velocity will tend to occur too late.

I do not claim that the transition length found in this work is more accurate than a factor of 2 . I do claim that since the physical model has a nonsingular solution and the rupture width is at least $\Delta x$, the trends and relations found in this work are meaningful.

Additional constraints can be put on the error of a solution by considering conservation principles. For the leapfrog equations there exist discrete analogues of momentum and energy. If boundary conditions on stress or velocity are imposed in a manner consistent with the internal differencing, then the sums over the grid of these discrete analogues agree precisely with the impulse and work, respectively, done at the boundaries.

At grid point $j$ and time step $n$ the analogue of momentum per unit mass is

$$
\frac{1}{2}\left(v_{j}^{n+1 / 2}+v_{j}^{n-1 / 2}\right)
$$

and the analogue of energy per unit mass is

$$
\sigma_{0}= \pm \rho c_{0} v_{0}
$$




$$
\frac{1}{2} v_{j}{ }^{n+1 / 2} v_{j}^{n-1 / 2}+\frac{1}{2}\left(E_{J+1 / 2}{ }^{n}+E_{J-1 / 2}{ }^{n}\right)
$$

where $E$ is the strain energy function. Note that a mode with alternating velocity from one time step to the next is not limited by this energy analogue and is subject to instability. All other modes, even those significantly dispersed, are constrained by energy. conservation. These conservation principles can be extended to two dimensions, nonuniform grids, and nonlinear materials [Trulio and Trigger, 1961; Trulio, 1964, 1966].

A numerical solution for a crack propagating with an abrupt stress drop can be quite noisy. One reason is simply that amplitudes of short-wavelength components are significant, and those components are dispersed. There is a second cause. With an abrupt stress drop in a numerical calculation, there is no energy absorption at the rupture front as at the singularity in the corresponding analytic solution. Amplitudes of long-wavelength components will be accurate, so that the energy that should be absorbed must go into shorter-wavelength components. Therefore the crack tip is a source of short-wavelength noise. Figure 8 shows that the slip-weakening model is not generating much noise, even with the rupture front as narrow as $\Delta x$.

Note added in proof. The question of the validity of spreading out a crack solution in space-time is equivalent to the question of superposition of solutions with prescribed plastic strain. Burridge and Knopoff [1964] and Nyland [1971] have shown that plastic strain is equivalent to distributed body force, and the validity of superposition follows immediately. The superposed solution will satisfy the equation of motion, consistent with the superposed plastic strain. At points where plastic strain is zero, Hooke's law will be satisfied. However, a given constitutive law relating plastic strain to stress history will not be satisfied both before and after superposition. If a superposed solution satisfies a desired constitutive relation, then it is the desired solution, even though it is formed from solutions satisfying different constitutive relations.

Acknowledgments. I am grateful for fruitful discussions with Paul Richards, Keiiti Aki, and William Joyner. Publication approved by the Director, U.S. Geological Survey.

\section{REFERENCES}

Alford, R. M., K. R. Kelly, and D. M. Boore, Accuracy of finitedifference modeling of the acoustic wave equation, Geophysics, 39 , 834-842, 1974.
Andrews, D. J., A numerical study of tectonic stress release by underground explosions, Bull. Seismol. Soc. Amer., 63, 1375-1391, 1973.

Andrews, D. J., Rupture propagation with finite stress in antiplane strain, J. Geophys. Res., 81, 3575, 1976.

Burridge, R., Admissible speeds for plane-strain self-similar shear cracks with friction but lacking cohesion, Geophys. J. Roy. Astron. Soc., 35, 439-455, 1973.

Burridge, R., and L. Knopoff, Body force equivalents of seismic dislocations, Bull. Seismol. Soc. Amer., 54, 1875-1888, 1964.

Burridge, R., and J. R. Willis, The self-similar problem of the expanding elliptical crack in an anisotropic solid, Proc. Cambridge Phil. Soc., 66, 443-468, 1969.

Fossum, A. F., and L. B. Freund, Nonuniformly moving shear crack model of a shallow focus earthquake mechanism, J. Geophys. Res., 80, 3343-3347, 1975.

Freund, L. B., Energy flux into the tip of an extending crack in an elastic solid, J. Elasticity, 2, 341-348, 1972.

Hamano, Y., Dependence of rupture time history on the heterogeneous distribution of stress and strength on the fault plane (abstract), Eos Trans. $A G U, 55,352,1974$.

Ida, Y., Cohesive force across the tip of a longitudinal shear crack and Griffith's specific surface energy, J. Geophys. Res., 77, 3796-3805, 1972.

Kostrov, B. V., The theory of the focus for tectonic earthquakes, Izv. Acad. Sci. USSR Phys. Solid Earth. Engl. Transl., no. 4, 258-267, 1970.

Madariaga, R., Dynamics of an expanding circular fault, Bull. Seismol. Soc. Amer., 66, in press, 1976.

Nyland, E., Body force equivalents as sources of anelastic processes, Can. J. Earth Sci., 8, 1184-1189, 1971.

Richards, P. G., Dynamic motions near an earthquake fault: A threedimensional solution, Bull. Seismol. Soc. Amer., 66, 1-32, 1976.

Richtmyer, R. D., and K. W. Morton, Difference Methods for InitialValue Problems, p. 262, Interscience, New York, 1967.

Starr, A. T., Slip in a crystal and rupture in a solid due to shear, Proc. Cambridge Phil. Soc., 24, 489-500, 1928.

Trulio, J. G., Studies of finite difference techniques for continuum mechanics, Tech. Rep. WL TDR-64-72, Air Force Weapons Lab., Kirtland Air Force Base, N. Mex., 1964.

Trulio, J. G., Theory and structure of the Afton codes, Tech. Rep. AFWL-TR-66-19. Air Force Weapons Lab., Kirtland Air Force Base, N. Mex., 1966.

Trulio, J. G., and K. R. Trigger, Numerical solution of the onedimensional Lagrangian hydrodynamic equations, Tech. Rep. UCRL 6267, Lawrence Radiat. Lab., Livermore, Calif., 1961.

Zemanian, A. H., Distribution Theory and Transform Analysis, pp. 1-14, 36, 72, 122-137, McGraw-Hill, New York, 1965.
(Received March 31, 1976; revised June 23, 1976; accepted July 1, 1976.) 